D.T. Wickramasinghe, L. Ferrario, and G.V. Bicknell, eds.

\title{
Numerical Simulations Of Advective Flows Around Black Holes
}

\author{
Sandip K. Chakrabarti ${ }^{1}$, D. Ryu ${ }^{2}$, D. Molteni ${ }^{3}$, H. Sponholz ${ }^{4}$, G. \\ Lanzafame $^{5}$, G. Eggum ${ }^{6}$ \\ 1. Tata Institute Of Fundamental Research, Mumbai, 400005, INDIA \\ 2. Chungnam National University, Daejeon, SOUTH KOREA \\ 3. Istitut di Fisica, Via Archirafi 36, 90123 Palermo, ITALY \\ 4. University of Kentucky, Lexington, USA \\ 5. Osservatorio di Catania, Catania, Sicily, ITALY \\ 6. Los Alamos National Laboratory, Los Alamos, NM, USA
}

Observational results of compact objects are best understood using advective accretion flows (Chakrabarti, 1996, 1997). We present here the results of numerical simulations of all possible types of such flows.

Two parameter (specific energy $\mathcal{E}$ and specific angular momentum $\lambda$ ) space of solutions of inviscid advective flow is classified into 'SA' (shocks in accretion), 'NSA' (no shock in accretion), ' $\mathrm{I}$ ' (inner sonic point only), ' $\mathrm{O}$ ' (outer sonic point only) etc. (Fig. 1 of Chakrabarti, 1997 and references therein). Fig. 1a shows examples of solutions (Molteni, Ryu \& Chakrabarti, 1996; Eggum, in preparation) from 'SA', ' $\mathrm{I}$ ' and ' $\mathrm{O}$ ' regions where we superpose analytical (solid) and numerical simulations (short dashed curve is with SPH code and medium dashed curve is with TVD code; very long dashed curve is with explicit/implicit code). The agreement is excellent. In presence of cooling effects, shocks from 'SA' oscillate (Fig. 1b) when the cooling timescale roughly agrees with postshock infall time scale (Molteni, Sponholz \& Chakrabarti, 1996). The solid, long dashed and short dashed curves are drawn for $T^{1 / 2}$ (bremsstrahlung), $T^{0.4}$ and $T^{0.75}$
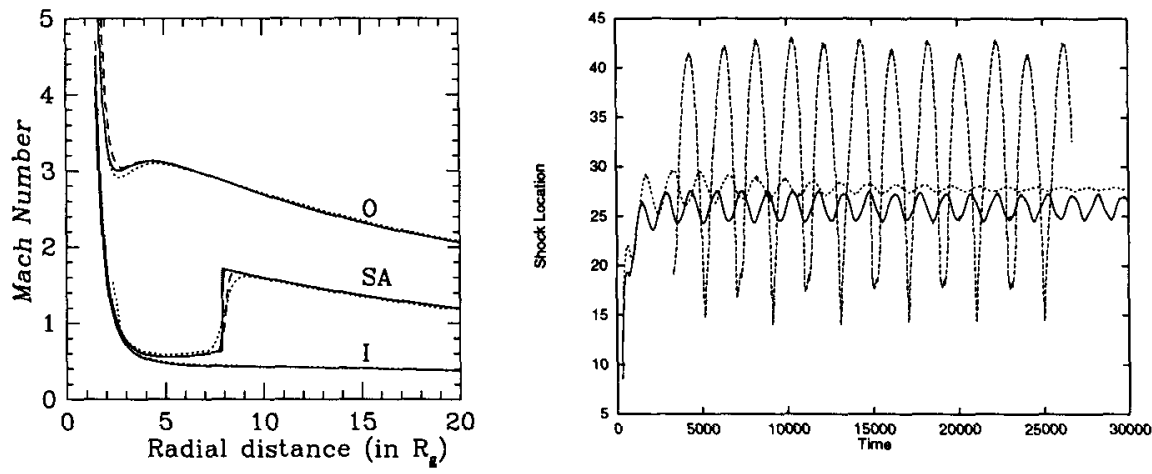

Figure 1. (a) Numerical simulations and theoretical calculations of shock-free (O, I) and shocked (SA) flows. (b) Oscillation of shocks when cooling effects are included. 
cooling laws respectively. In the absence of steady shock solutions, shocks for parameters from 'NSA' oscillate (Fig. 2) even in the absence of viscosity (Ryu et al. 1997). The oscillation frequency and amplitude roughly agree with those of quasi-periodic oscillation of black hole candidates. When the flow starts from a cool Keplerian disk, it simply becomes sub-Keplerian before it enters through the horizon. Fig. 3a shows this behaviour where the ratio of $\lambda / \lambda_{\text {Keplerian }}$ is plotted. When the flow deviates from a hot Keplerian disk, it may develop a standing shock as well (Fig. 3b) (Molteni et al. 1996).

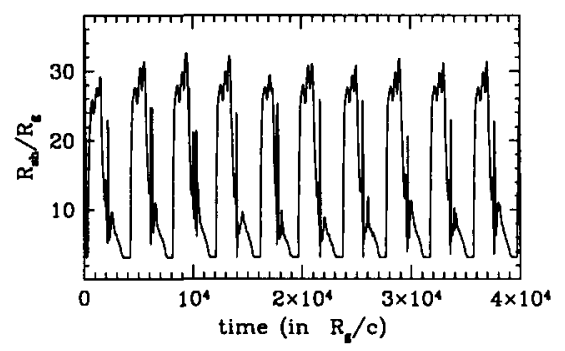

Figure 2. Oscillation of shock location when the flow has two sonic points but a steady shock condition is not satisfied.
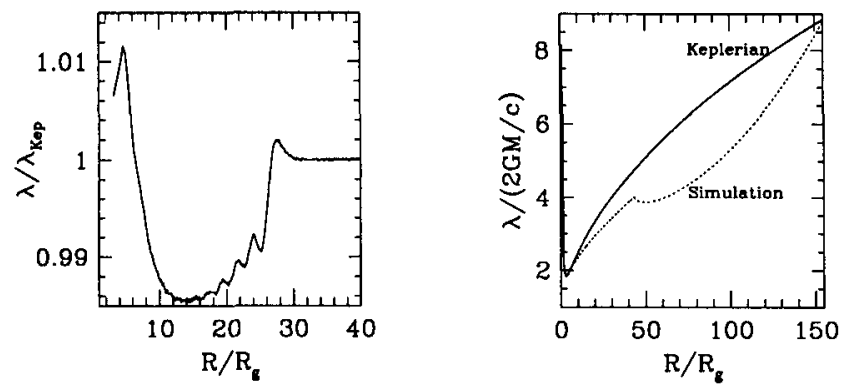

Figure 3. (a) Ratio $\lambda_{\text {disk }} / \lambda_{\text {Kep }}$ as obtained from a 1D numerical simulation. (b) Another 1D simulation which shows formation of subKeplerian flows and shocks from a hot Keplerian disk.

\section{References}

Chakrabarti, S.K. 1996, Phys. Rep., 266, 229 (Chapter V)

Chakrabarti, S.K. 1997 (this volume)

Molteni, D., Chakrabarti, S.K., Bisikalo, D. \& Kuznetzov, O. 1996, MNRAS.

Molteni, D., Ryu, D. \& Chakrabarti, S.K. 1996, ApJ(Oct. 10th)

Molteni, D., Sponholz, H., \& Chakrabarti, S.K. 1996, ApJ, 457, 805

Ryu, D., \& Chakrabarti, S.K., \& Molteni, D. 1997, ApJ(Jan. 1st) 